\title{
Hamilton-Souplet-Zhang's Gradient Estimates for Two Types of Nonlinear Parabolic Equations under the Ricci Flow
}

\author{
Guangyue Huang ${ }^{1}$ and Bingqing $\mathrm{Ma}^{2}$ \\ ${ }^{1}$ College of Mathematics and Information Science, Henan Normal University, Xinxiang, Henan 453007, China \\ ${ }^{2}$ Henan Engineering Laboratory for Big Data Statistical Analysis and Optimal Control, College of Mathematics and \\ Information Science, Henan Normal University, Xinxiang, Henan 453007, China
}

Correspondence should be addressed to Bingqing Ma; bqma@henannu.edu.cn

Received 10 November 2015; Accepted 29 December 2015

Academic Editor: Igor E. Verbitsky

Copyright (C) 2016 G. Huang and B. Ma. This is an open access article distributed under the Creative Commons Attribution License, which permits unrestricted use, distribution, and reproduction in any medium, provided the original work is properly cited.

We consider gradient estimates for two types of nonlinear parabolic equations under the Ricci flow: one is the equation $u_{t}=$ $\Delta u+a u \log u+b u$ with $a, b$ being two real constants; the other is $u_{t}=\Delta u+\lambda u^{\alpha}$ with $\lambda, \alpha$ being two real constants. By a suitable scaling for the above two equations, we obtain Hamilton-Souplet-Zhang-type gradient estimates.

\section{Introduction}

Since the nonlinear parabolic equation

$$
u_{t}=\Delta u+a u \log u+b u
$$

on a given Riemannian manifold is related to gradient Ricci solitons which are self-similar solutions to the Ricci flow, many attentions are paid to the study on gradient estimates for (1); for example, see [1-7]. Here $a, b$ in (1) are two real constants. Clearly, a heat equation

$$
u_{t}=\Delta u
$$

is a special case of (1) when $a=b=0$. Hence many known results on heat equations are generalized to the nonlinear parabolic equation (1). For gradient estimates of solutions to (1) of Li-Yau type, Davies type, Hamilton type, and Li-Xu type on a given Riemannian manifold, we refer to $[1,3,7,8]$ and the references therein. In a recent paper [9], Dung and Khanh obtained Hamilton-Souplet-Zhang-type gradient estimates on a given Riemannian manifold for (1). On a family of Riemannian metrics $g(t)$ evolving from the Ricci flow

$$
\frac{\partial}{\partial t} g_{i j}=-2 R_{i j}
$$

Hsu in [10] obtained Li-Yau-type gradient estimates of (1).
In [11], generalizing Hamilton's estimate in [12], Souplet and Zhang proved the following result.

Theorem A (see [11]). Let $\left(M^{n}, g\right)$ be an $n$-dimensional Riemannian manifold with $\operatorname{Ric}\left(M^{n}\right) \geq-k$, where $k$ is a nonnegative constant. Suppose that $u$ is a positive solution to (2) in $Q_{R, T}=\left\{(x, t) \mid x \in M, d\left(x, x_{0}, t\right)<R, t \in[0, T]\right\}$ with $u \leq A$. Then in $Q_{R, T}$,

$$
\frac{|\nabla u|}{u} \leq C\left(\sqrt{k}+\frac{1}{R}+\frac{1}{\sqrt{T}}\right)\left(1+\log \frac{A}{u}\right)
$$

where constant $C$ depends only on the dimension $n$.

The key to prove Theorem A of Souplet and Zhang is the scaling $u \rightarrow \widetilde{u}=u / A$. After this scaling, (2) becomes the following heat equation with respect to $\tilde{u}$ :

$$
\tilde{u}_{t}=\Delta \tilde{u}
$$

since the heat equation is linear. Under this case, we obtain that $0<\tilde{u} \leq 1$. Inspired by this method, in this paper, we also adopt the similar scaling method by $u \rightarrow \tilde{u}=u / A$ to study the nonlinear parabolic equation (1). By the scaling, we can derive from (1) the following analogous equation:

$$
\tilde{u}_{t}=\Delta \tilde{u}+a \tilde{u} \log \tilde{u}+\tilde{b} \tilde{u},
$$


where constant $\widetilde{b}$ satisfies $\widetilde{b}=b+a \log A$. That is, we only need to study the nonlinear equation (6) with $0<\tilde{u} \leq 1$.

Our first result is the following Hamilton-Souplet-Zhangtype gradient estimates of the nonlinear equation (1) under the Ricci flow.

Theorem 1. Let $M$ be a complete Riemannian manifold with a family of Riemannian metrics $g(t)$ evolving from the Ricci flow (3). Suppose that $u$ is a positive solution to (1) in

$$
B_{R, T}=\left\{(x, t) \mid x \in M, d\left(x, x_{0}, t\right)<R, t \in[0, T]\right\}
$$

with $|\mathrm{Ric}| \leq k$ for some positive constant $k$ and $u \leq A$. Then there exists a constant $C$ depending only on the dimension of $M$ such that

$$
\frac{|\nabla u|}{u} \leq C\left(\frac{1}{R}+\frac{1}{\sqrt{T}}+\sqrt{k}+\sqrt{M_{a, b}}\right)\left(1+\log \frac{A}{u}\right)
$$

for all $(x, t) \in B_{R / 2, T}$ with $t \neq 0$, where $M_{a, b}=\max \{0, a(1+$ $\log A)+b\}$.

The study of Li-Yau-type estimates of the following nonlinear parabolic equation:

$$
u_{t}=\Delta u+\lambda u^{\alpha}
$$

where $\lambda, \alpha$ are two real constants, can be traced back to Li [13]. Later, for $0<\alpha<1$, Zhu in [14] obtained Hamilton-SoupletZhang-type gradient estimates of (9) on a given Riemannian manifold. On gradient estimates of the elliptic case of (9), see $[15,16]$. A natural subject is to study Hamilton-SoupletZhang-type gradient estimates of the nonlinear equation (9) under the Ricci flow. Our second result is the following.

Theorem 2. Let $M$ be a complete Riemannian manifold with a family of Riemannian metrics $g(t)$ evolving from the Ricci flow (3). Suppose that $u$ is a positive solution to (9) in

$$
B_{R, T}=\left\{(x, t) \mid x \in M, d\left(x, x_{0}, t\right)<R, t \in[0, T]\right\}
$$

with $|\mathrm{Ric}| \leq k$ for some positive constant $k$ and $u \leq B$. Then there exists a constant $C$ depending only on the dimension of $M$ such that

(1) if $\alpha \geq 1$, then

$$
\frac{|\nabla u|}{u} \leq C\left(\frac{1}{R}+\frac{1}{\sqrt{T}}+\sqrt{k}+\sqrt{M_{\lambda} \alpha}\right)\left(1+\log \frac{B}{u}\right),
$$

for all $(x, t) \in B_{R / 2, T / 2}$ with $t \neq 0$, where $M_{\lambda}=$ $\max \left\{0, \lambda B^{\alpha-1}\right\}$;

(2) if $\alpha \leq 0$, then

$$
\begin{aligned}
\frac{|\nabla u|}{u} \leq & C\left(\frac{1}{R}+\frac{1}{\sqrt{T}}+\sqrt{k}+\sqrt{M_{\lambda}(-\alpha+1) u_{\min }^{\alpha-1}}\right) \\
& \cdot\left(1+\log \frac{B}{u}\right)
\end{aligned}
$$

for all $(x, t) \in B_{R / 2, T}$ with $t \neq 0$, where $M_{\lambda}=$ $\max \{0,-\lambda\}$;
(3) if $\alpha \in(0,1)$, then

$$
\begin{aligned}
& \frac{|\nabla u|}{u} \leq C\left(\frac{1}{R}+\frac{1}{\sqrt{T}}+\sqrt{k}+\sqrt{|\lambda| u_{\min }^{\alpha-1}}\right)\left(1+\log \frac{B}{u}\right) \\
& \quad \text { for all }(x, t) \in B_{R / 2, T} \text { with } t \neq 0 .
\end{aligned}
$$

Remark 3. Taking $a=b=0$ in (8), we obtain the estimate (16) of Theorem 2.2 in [17] with respect to the heat equation under the Ricci flow. Hence, our estimates in Theorem 1 extend Bailesteanu, Cao, and Pulemotov's Theorem 2.2.

Remark 4. There are many studies on gradient estimates of the heat equation (2) under geometric flows; we refer to [18, 19] among others.

\section{Proof of Theorem 1}

In order to prove our Theorem 1, we first give a lemma which will play an important role in the proof.

Lemma 5. Let $M$ be a complete Riemannian manifold with a family of Riemannian metrics $g(t)$ evolving from the Ricci flow (3). Let $u$ be a positive solution to (1) with $u \leq A$. Denoting by $\tilde{u}=u / A, f=\log \tilde{u} \leq 0$ and $w=|\nabla \log (1-f)|^{2}$. Then, it holds that

$$
\begin{aligned}
\left(\Delta-\partial_{t}\right) w \geq & \frac{2 f}{1-f} \nabla f \nabla w+2(1-f) w^{2} \\
& -\frac{2(a+B)}{1-f} w
\end{aligned}
$$

where $B=b+a \log A$.

Proof. Under the scaling $u \rightarrow \widetilde{u}=u / A$, we have $0<\widetilde{u} \leq 1$. From (1), we obtain that $\tilde{u}$ satisfies the following equation:

$$
\tilde{u}_{t}=\Delta \widetilde{u}+a \tilde{u} \log \tilde{u}+B \tilde{u}
$$

where constant $B$ satisfies $B=b+a \log A$. Let $f=\log \widetilde{u} \leq 0$ and $w=|\nabla \log (1-f)|^{2}=|\nabla f|^{2} /(1-f)^{2}$. Then we have

$$
f_{t}=\Delta f+|\nabla f|^{2}+a f+B
$$

Using (3), we can obtain

$$
\left(|\nabla f|^{2}\right)_{t}=2 R_{i j} f_{i} f_{j}+2 f_{i}\left(f_{t}\right)_{i} .
$$

By the definition of $w$, we have

$$
\begin{aligned}
w_{t}= & \frac{2}{(1-f)^{2}}\left[R_{i j} f_{i} f_{j}+f_{i}\left(f_{t}\right)_{i}\right]+\frac{2}{(1-f)^{3}} f_{j}^{2} f_{t} \\
= & \frac{2}{(1-f)^{2}}\left[R_{i j} f_{i} f_{j}+f_{i} f_{j j i}+2 f_{i j} f_{i} f_{j}+a f_{i}^{2}\right] \\
& +\frac{2}{(1-f)^{3}} f_{j}^{2}\left(f_{i i}+f_{i}^{2}+a f+B\right) .
\end{aligned}
$$


On the other hand,

$$
\begin{aligned}
\Delta w= & \frac{6}{(1-f)^{4}} f_{i}^{2} f_{j}^{2}+\frac{2}{(1-f)^{3}} f_{i i} f_{j}^{2} \\
& +\frac{8}{(1-f)^{3}} f_{j i} f_{i} f_{j}+\frac{2}{(1-f)^{2}} f_{j i}^{2} \\
& +\frac{2}{(1-f)^{2}} f_{j} f_{j i i} \\
= & \frac{6}{(1-f)^{4}} f_{i}^{2} f_{j}^{2}+\frac{2}{(1-f)^{3}} f_{i i} f_{j}^{2} \\
& +\frac{8}{(1-f)^{3}} f_{j i} f_{i} f_{j}+\frac{2}{(1-f)^{2}} f_{j i}^{2} \\
& +\frac{2}{(1-f)^{2}} f_{j} f_{i i j}+\frac{2}{(1-f)^{2}} R_{i j} f_{i} f_{j},
\end{aligned}
$$

where, in the second equality, we used the Ricci formula

$$
f_{j i i}=f_{i j i}=f_{i i j}+R_{i j} f_{i} .
$$

By the formulas (18) and (19), we arrive at

$$
\begin{aligned}
\left(\Delta-\partial_{t}\right) w= & \frac{2}{(1-f)^{2}} f_{j i}^{2}+\frac{8}{(1-f)^{3}} f_{j i} f_{i} f_{j} \\
& -\frac{4}{(1-f)^{2}} f_{i j} f_{i} f_{j}+\frac{6}{(1-f)^{4}} f_{i}^{2} f_{j}^{2} \\
& -\frac{2}{(1-f)^{3}} f_{i}^{2} f_{j}^{2}-\frac{2(a+B)}{1-f} \frac{f_{i}^{2}}{(1-f)^{2}} .
\end{aligned}
$$

Note that

$$
\nabla f \nabla w=\frac{2}{(1-f)^{3}} f_{i}^{2} f_{j}^{2}+\frac{2}{(1-f)^{2}} f_{j i} f_{i} f_{j} .
$$

Therefore, (21) can be written as

$$
\begin{aligned}
\left(\Delta-\partial_{t}\right) w= & \frac{2}{(1-f)^{2}} f_{j i}^{2}+\frac{4}{(1-f)^{3}} f_{j i} f_{i} f_{j} \\
& -\frac{4}{(1-f)^{2}} f_{i j} f_{i} f_{j}+\frac{2}{1-f} \nabla f \nabla w \\
& +2 \frac{|\nabla f|^{4}}{(1-f)^{4}}-2 \frac{|\nabla f|^{4}}{(1-f)^{3}} \\
& -\frac{2(a+B)}{1-f} \frac{|\nabla f|^{2}}{(1-f)^{2}}
\end{aligned}
$$

$$
\begin{aligned}
= & \frac{2}{(1-f)^{2}} f_{j i}^{2}+\frac{4}{(1-f)^{3}} f_{j i} f_{i} f_{j} \\
& -2 \nabla f \nabla w+\frac{2}{1-f} \nabla f \nabla w+2 \frac{|\nabla f|^{4}}{(1-f)^{4}} \\
& +2 \frac{|\nabla f|^{4}}{(1-f)^{3}}-\frac{2(a+B)}{1-f} \frac{|\nabla f|^{2}}{(1-f)^{2}} \\
= & \frac{2}{(1-f)^{2}}\left(f_{j i}+\frac{1}{1-f} f_{i} f_{j}\right)^{2} \\
& +\frac{2 f}{1-f} \nabla f \nabla w+2 \frac{|\nabla f|^{4}}{(1-f)^{3}} \\
& -\frac{2(a+B)}{1-f} \frac{|\nabla f|^{2}}{(1-f)^{2}} \\
\geq & \frac{2 f}{1-f} \nabla f \nabla w+2(1-f) w^{2} \\
& -\frac{2(a+B)}{1-f} w .
\end{aligned}
$$

Then, the desired estimate (14) follows.

Proof of Theorem 1. Let $\psi=\psi(x, t)$ be a smooth cutoff function supported in $B_{R, T}$ which satisfies the following properties (see page 13 in [20] or Lemma 2.1 in [17]):

(1) $\psi=\psi\left(d\left(x, x_{0}, t\right), t\right) \equiv \psi(r, t) ; \psi(x, t)=1$ in $B_{R / 2, T / 2}$ and $0 \leq \psi \leq 1$.

(2) $\psi$ is decreasing as a radial function in the spatial variables.

(3) $|\partial \psi / \partial r| \leq\left(C_{\gamma} / R\right) \psi^{\gamma}$ and $\left|\partial^{2} \psi / \partial r^{2}\right| \leq\left(C_{\gamma} / R^{2}\right) \psi^{\gamma}$ for every $\gamma \in(0,1)$.

(4) $|\partial \psi / \partial t| \leq(C / T) \psi^{1 / 2}$.

We use (14) to conclude that

$$
\begin{aligned}
\left(\Delta-\partial_{t}\right)(\psi w)= & \psi\left(\Delta-\partial_{t}\right) w+w\left(\Delta-\partial_{t}\right) \psi \\
& +2 \nabla \psi \nabla w \\
= & \psi\left(\Delta-\partial_{t}\right) w+w\left(\Delta-\partial_{t}\right) \psi \\
& +2 \frac{\nabla \psi}{\psi} \nabla(\psi w)-2 w \frac{|\nabla \psi|^{2}}{\psi} \\
\geq & \frac{2 f}{1-f}[\nabla f \nabla(\psi w)-w \nabla f \nabla \psi] \\
& +2(1-f) \psi w^{2}-\frac{2(a+B)}{1-f} \psi w
\end{aligned}
$$




$$
\begin{aligned}
& +w\left(\Delta-\partial_{t}\right) \psi+2 \frac{\nabla \psi}{\psi} \nabla(\psi w) \\
& -2 w \frac{|\nabla \psi|^{2}}{\psi} .
\end{aligned}
$$

Now we let $\left(x_{1}, t_{1}\right)$ be a maximum point of $\psi w$ in the closure of $B_{R, T}$, and where $\psi w>0$ (otherwise the proof is trivial). Then at the point $\left(x_{1}, t_{1}\right)$, we have $\Delta(\psi w) \leq 0,(\psi w)_{t} \geq 0$, and $\nabla(\psi w)=0$. Thus, from (24), we deduce

$$
\begin{aligned}
2(1-f) \psi w^{2} \leq & \frac{2 f}{1-f} w \nabla f \nabla \psi+\frac{2(a+B)}{1-f} \psi w \\
& -w\left(\Delta-\partial_{t}\right) \psi+2 w \frac{|\nabla \psi|^{2}}{\psi}
\end{aligned}
$$

at $\left(x_{1}, t_{1}\right)$. Next, we will find an upper bound for each term of the right-hand side of (25). By the Cauchy inequality, we have

$$
\begin{aligned}
\frac{2 f}{1-f} w \nabla f \nabla \psi & \leq 2|f| w^{3 / 2}|\nabla \psi| \\
& =2\left[\psi(1-f) w^{2}\right]^{3 / 4} \frac{f|\nabla \psi|}{[\psi(1-f)]^{3 / 4}} \\
& \leq(1-f) \psi w^{2}+C \frac{1}{R^{4}} \frac{f^{4}}{(1-f)^{3}} .
\end{aligned}
$$

It has been shown in [20] (see formulas (3.30), (3.32), and (3.34) in [20]) that

$$
\begin{aligned}
w \frac{|\nabla \psi|^{2}}{\psi} & \leq \frac{1}{8} \psi w^{2}+C \frac{1}{R^{4}} \\
-(\Delta \psi) w & \leq \frac{1}{8} \psi w^{2}+C \frac{1}{R^{4}}+C \frac{k}{R^{2}} \\
\left|\frac{\partial \psi}{\partial t}\right| w & \leq \frac{1}{8} \psi w^{2}+C \frac{1}{T^{2}}+C k^{2} .
\end{aligned}
$$

Putting (26)-(27) into (25), we obtain

$$
\begin{aligned}
(1-f) \psi w^{2} \leq & C \frac{1}{R^{4}} \frac{f^{4}}{(1-f)^{3}}+\frac{2(a+B)}{1-f} \psi w \\
& +\frac{1}{2} \psi w^{2}+C \frac{1}{R^{4}}+C \frac{1}{T^{2}}+C k^{2} .
\end{aligned}
$$

Since $f \leq 0$, the inequality (28) implies

$$
\begin{aligned}
& \psi w^{2} \leq C\left(\frac{1}{R^{4}} \frac{f^{4}}{(1-f)^{4}}+\frac{1}{R^{4}}+\frac{1}{T^{2}}+k^{2}\right. \\
& \left.+(\max \{0, a+B\})^{2}\right)=C\left(\frac{1}{R^{4}} \frac{f^{4}}{(1-f)^{4}}+\frac{1}{R^{4}}\right. \\
& \left.+\frac{1}{T^{2}}+k^{2}+M_{a, b}^{2}\right) .
\end{aligned}
$$

Therefore, we deduce that, for any $(x, t) \in B_{R, T}$,

$$
\begin{aligned}
\left(\psi^{2} w^{2}\right)(x, t) & \leq\left(\psi w^{2}\right)\left(x_{1}, t_{1}\right) \\
& \leq C\left(\frac{1}{R^{4}}+\frac{1}{T^{2}}+k^{2}+M_{a, b}^{2}\right)
\end{aligned}
$$

from $f^{4} /(1-f)^{4}<1$. Noticing $\psi(x, t)=1$ in $B_{R / 2, T / 2}$ and $w=|\nabla f|^{2} /(1-f)^{2}$, we have the fact that

$$
\frac{|\nabla f|}{1-f} \leq C\left(\frac{1}{R}+\frac{1}{\sqrt{T}}+\sqrt{k}+\sqrt{M_{a, b}}\right)
$$

holds at any $(x, t) \in B_{R / 2, T}$, which shows that

$$
\frac{|\nabla u|}{u} \leq C\left(\frac{1}{R}+\frac{1}{\sqrt{T}}+\sqrt{k}+\sqrt{M_{a, b}}\right)\left(1+\log \frac{A}{u}\right) .
$$

We complete the proof of the estimate (8) in Theorem 1.

\section{Proof of Theorem 2}

As in the proof of Theorem 1, we first give a key lemma.

Lemma 6. Let $M$ be a complete Riemannian manifold with a family of Riemannian metrics $g(t)$ evolving from the Ricci flow (3). Let $u$ be a positive solution to (9) with $u \leq B$. Denoting by $\widetilde{u}=u / B, f=\log \widetilde{u} \leq 0$ and $w=|\nabla \log (1-f)|^{2}$. Then, it holds that

$$
\begin{aligned}
\left(\Delta-\partial_{t}\right) w \geq & \frac{2 f}{1-f}\langle\nabla f, \nabla w\rangle+2(1-f) w^{2} \\
& -2 \tilde{\lambda}\left(\alpha-\frac{-f}{1-f}\right) e^{(\alpha-1) f} w,
\end{aligned}
$$

where $\tilde{\lambda}=\lambda B^{\alpha-1}$.

Proof. By the scaling $u \rightarrow \widetilde{u}=u / B$, we have $0<\widetilde{u} \leq 1$. Therefore, we obtain from (9) that $\tilde{u}$ satisfies

$$
\tilde{u}_{t}=\Delta \tilde{u}+\tilde{\lambda} \tilde{u}^{\alpha}
$$

where $\tilde{\lambda}=\lambda B^{\alpha-1}$. Let $f=\log \tilde{u} \leq 0$ and

$$
w=|\nabla \log (1-f)|^{2}
$$

Then, the function $f$ satisfies

$$
f_{t}=\Delta f+|\nabla f|^{2}+\tilde{\lambda} e^{(\alpha-1) f} .
$$

Using (3) again, one has

$$
\left(|\nabla f|^{2}\right)_{t}=2 R_{i j} f_{i} f_{j}+2 f_{i}\left(f_{t}\right)_{i} .
$$


It follows from (35) that

$$
\begin{aligned}
w_{t} & =\frac{2}{(1-f)^{2}}\left[R_{i j} f_{i} f_{j}+f_{i}\left(f_{t}\right)_{i}\right]+\frac{2}{(1-f)^{3}} f_{j}^{2} f_{t} \\
& =\frac{2}{(1-f)^{2}}\left[R_{i j} f_{i} f_{j}+f_{i} f_{j j i}+2 f_{i j} f_{i} f_{j}\right. \\
& \left.+\widetilde{\lambda}(\alpha-1) e^{(\alpha-1) f} f_{i}^{2}\right]+\frac{2}{(1-f)^{3}} f_{j}^{2}\left(f_{i i}+f_{i}^{2}\right. \\
& \left.+\widetilde{\lambda} e^{(\alpha-1) f}\right) .
\end{aligned}
$$

Similarly, by the Ricci formula, we obtain

$$
\begin{aligned}
\Delta w= & \frac{6}{(1-f)^{4}} f_{i}^{2} f_{j}^{2}+\frac{2}{(1-f)^{3}} f_{i i} f_{j}^{2} \\
& +\frac{8}{(1-f)^{3}} f_{j i} f_{i} f_{j}+\frac{2}{(1-f)^{2}} f_{j i}^{2} \\
& +\frac{2}{(1-f)^{2}} f_{j} f_{i i j}+\frac{2}{(1-f)^{2}} R_{i j} f_{i} f_{j} .
\end{aligned}
$$

Thus, we derive from (38) and (39) the following:

$$
\begin{aligned}
\left(\Delta-\partial_{t}\right) w \\
=\frac{2}{(1-f)^{2}} f_{j i}^{2}+\left[\frac{6}{(1-f)^{4}}-\frac{2}{(1-f)^{3}}\right] f_{i}^{2} f_{j}^{2} \\
+\left[\frac{8}{(1-f)^{3}}-\frac{4}{(1-f)^{2}}\right] f_{j i} f_{i} f_{j} \\
\quad-2 \tilde{\lambda}\left[(\alpha-1) \frac{1}{(1-f)^{2}}+\frac{1}{(1-f)^{3}}\right] e^{(\alpha-1) f} f_{i}^{2} .
\end{aligned}
$$

Using the relationship

$$
\langle\nabla f, \nabla w\rangle=\frac{2}{(1-f)^{3}} f_{i}^{2} f_{j}^{2}+\frac{2}{(1-f)^{2}} f_{j i} f_{i} f_{j},
$$

(40) can be written as

$$
\begin{aligned}
(\Delta- & \left.\partial_{t}\right) w-\varepsilon\langle\nabla f, \nabla w\rangle \\
= & \frac{2}{(1-f)^{2}} f_{j i}^{2} \\
& +2\left[\frac{3}{(1-f)^{4}}-(1+\varepsilon) \frac{1}{(1-f)^{3}}\right] f_{i}^{2} f_{j}^{2} \\
& +2\left[\frac{4}{(1-f)^{3}}-(2+\varepsilon) \frac{1}{(1-f)^{2}}\right] f_{j i} f_{i} f_{j} \\
& -2 \tilde{\lambda}\left[(\alpha-1) \frac{1}{(1-f)^{2}}+\frac{1}{(1-f)^{3}}\right] e^{(\alpha-1) f} f_{i}^{2}
\end{aligned}
$$

where $\varepsilon=\varepsilon(f)$ is a function depending on $f$ which will be determined. Applying the inequality

$$
\begin{aligned}
& \frac{2}{(1-f)^{2}} f_{j i}^{2} \\
& +2\left[\frac{4}{(1-f)^{3}}-(2+\varepsilon) \frac{1}{(1-f)^{2}}\right] f_{j i} f_{i} f_{j} \\
& =\frac{2}{(1-f)^{2}}\left\{f_{j i}^{2}+\left[\frac{4}{1-f}-(2+\varepsilon)\right] f_{j i} f_{i} f_{j}\right\} \\
& \geq-\frac{1}{2(1-f)^{2}}\left[\frac{4}{1-f}-(2+\varepsilon)\right]^{2} f_{i}^{2} f_{j}^{2}
\end{aligned}
$$

into (42) gives

$$
\begin{aligned}
& \left(\Delta-\partial_{t}\right) w-\varepsilon\langle\nabla f, \nabla w\rangle \geq\left[-\frac{2}{(1-f)^{4}}\right. \\
& \left.+(6+2 \varepsilon) \frac{1}{(1-f)^{3}}-(2+\varepsilon)^{2} \frac{1}{2(1-f)^{2}}\right] f_{i}^{2} f_{j}^{2} \\
& -2 \tilde{\lambda}\left[(\alpha-1) \frac{1}{(1-f)^{2}}+\frac{1}{(1-f)^{3}}\right] e^{(\alpha-1) f} f_{i}^{2} \\
& =\frac{1}{(1-f)^{4}}\left\{-\frac{1}{2}(1-f)^{2} \varepsilon^{2}\right. \\
& -2\left[(1-f)^{2}-(1-f)\right] \varepsilon \\
& \left.-\left[2(1-f)^{2}-6(1-f)+2\right]\right\} f_{i}^{2} f_{j}^{2} \\
& -2 \tilde{\lambda}\left[(\alpha-1) \frac{1}{(1-f)^{2}}+\frac{1}{(1-f)^{3}}\right] e^{(\alpha-1) f} f_{i}^{2} .
\end{aligned}
$$

Taking

$$
\varepsilon=-2+\frac{2}{1-f}
$$

in (44), we derive

$$
\begin{aligned}
(\Delta & \left.-\partial_{t}\right) w-\frac{2 f}{1-f}\langle\nabla f, \nabla w\rangle \\
\geq & \frac{2}{(1-f)^{3}}|\nabla f|^{4} \\
& -2 \tilde{\lambda}\left[(\alpha-1) \frac{1}{(1-f)^{2}}+\frac{1}{(1-f)^{3}}\right] e^{(\alpha-1) f} f_{i}^{2} \\
= & 2(1-f) w^{2}-2 \tilde{\lambda}\left(\alpha-\frac{-f}{1-f}\right) e^{(\alpha-1) f} w .
\end{aligned}
$$

Thus, the desired estimate (33) is attained. 
Proof of Theorem 2. Let $\psi=\psi(x, t)$ be a smooth cutoff function supported in $B_{R, T}$ which is defined in Section 2. We use (33) to conclude that

$$
\begin{aligned}
\left(\Delta-\partial_{t}\right)(\psi w)= & \psi\left(\Delta-\partial_{t}\right) w+w\left(\Delta-\partial_{t}\right) \psi \\
& +2 \frac{\nabla \psi}{\psi} \nabla(\psi w)-2 w \frac{|\nabla \psi|^{2}}{\psi} \\
\geq & -2 \frac{-f}{1-f}[\nabla f \nabla(\psi w)-w \nabla f \nabla \psi] \\
& +2(1-f) \psi w^{2} \\
& -2 \tilde{\lambda}\left(\alpha-\frac{-f}{1-f}\right) e^{(\alpha-1) f} \psi w \\
& +w\left(\Delta-\partial_{t}\right) \psi+2 \frac{\nabla \psi}{\psi} \nabla(\psi w) \\
& -2 w \frac{|\nabla \psi|^{2}}{\psi} .
\end{aligned}
$$

Now we let $\left(x_{2}, t_{2}\right)$ be a maximum point of $\psi w$ in the closure of $B_{R, T}$, where $\psi w>0$ (otherwise the proof is trivial). Then at the point $\left(x_{2}, t_{2}\right)$, we have $\Delta(\psi w) \leq 0,(\psi w)_{t} \geq 0$, and $\nabla(\psi w)=0$. Thus, from (47), we deduce that

$$
\begin{aligned}
2(1-f) \psi w^{2} \leq & \frac{2 f}{1-f} w \nabla f \nabla \psi \\
& +2 \tilde{\lambda}\left(\alpha-\frac{-f}{1-f}\right) e^{(\alpha-1) f} \psi w \\
& -w\left(\Delta-\partial_{t}\right) \psi+2 w \frac{|\nabla \psi|^{2}}{\psi}
\end{aligned}
$$

at $\left(x_{2}, t_{2}\right)$.

Case One $(\alpha \geq 1)$. Note that

$$
\frac{-f}{1-f}=1-\frac{1}{1-f} \in(0,1) \text {. }
$$

In this case, we have $\alpha-(-f /(1-f))>0$ and $e^{(\alpha-1) f} \in(0,1)$. Hence

$$
\begin{aligned}
& 2 \tilde{\lambda}\left(\alpha-\frac{-f}{1-f}\right) e^{(\alpha-1) f} \psi w \\
& \quad \leq C \frac{M_{\lambda}^{2}}{1-f}\left(\alpha-\frac{-f}{1-f}\right)^{2}+\frac{1}{16}(1-f) \psi w^{2},
\end{aligned}
$$

where $M_{\lambda}=\max \{0, \tilde{\lambda}\}$. Putting (50), (26)-(27) into (48), we obtain

$$
\begin{aligned}
& (1-f) \psi w^{2} \leq C\left[\frac{1}{R^{4}} \frac{f^{4}}{(1-f)^{3}}\right. \\
& \left.+\frac{M_{\lambda}^{2}}{1-f}\left(\alpha-\frac{-f}{1-f}\right)^{2}+\frac{1}{R^{4}}+\frac{1}{T^{2}}+k^{2}\right] .
\end{aligned}
$$

Therefore, we deduce, for any $(x, t) \in B_{R, T}$, that

$$
\begin{aligned}
\left(\psi^{2} w^{2}\right)(x, t) & \leq\left(\psi w^{2}\right)\left(x_{1}, t_{1}\right) \\
& \leq C\left(\frac{1}{R^{4}}+\frac{1}{T^{2}}+k^{2}+M_{\lambda}^{2} \alpha^{2}\right)
\end{aligned}
$$

from $f^{2} /(1-f)^{2}<1$. Noticing $\psi(x, t)=1$ in $B_{R / 2, T / 2}$ and $w=|\nabla f|^{2} /(1-f)^{2}$, we have the fact that

$$
\frac{|\nabla f|}{1-f} \leq C\left(\frac{1}{R}+\frac{1}{\sqrt{T}}+\sqrt{k}+\sqrt{M_{\lambda} \alpha}\right)
$$

holds at any $(x, t) \in B_{R / 2, T}$, which shows that

$$
\frac{|\nabla u|}{u} \leq C\left(\frac{1}{R}+\frac{1}{\sqrt{T}}+\sqrt{k}+\sqrt{M_{\lambda} \alpha}\right)\left(1+\log \frac{B}{u}\right) .
$$

Case Two $(\alpha \leq 0)$. In this case, we have $\alpha-(-f /(1-f))<0$ and $e^{(\alpha-1) f}>1$. Hence

$$
\begin{aligned}
2 \tilde{\lambda}\left(\alpha-\frac{-f}{1-f}\right) e^{(\alpha-1) f} \psi w \\
\leq C \frac{\widetilde{M}_{\lambda}^{2}}{1-f}\left(\alpha-\frac{-f}{1-f}\right)^{2} \widetilde{u}_{\min }^{2(\alpha-1)} \\
\quad+\frac{1}{16}(1-f) \psi w^{2},
\end{aligned}
$$

where $\widetilde{M}_{\lambda}=\max \{0,-\widetilde{\lambda}\}$. Similarly, putting (55), (26)-(27) into (48), we obtain

$$
\begin{aligned}
(1-f) \psi w^{2} & \leq C\left[\frac{1}{R^{4}} \frac{f^{4}}{(1-f)^{3}}\right. \\
& \left.+\frac{\widetilde{M}_{\lambda}^{2}}{1-f}\left(\alpha-\frac{-f}{1-f}\right)^{2} \widetilde{u}_{\min }^{2(\alpha-1)}+\frac{1}{R^{4}}+\frac{1}{T^{2}}+k^{2}\right] .
\end{aligned}
$$

Thus, we can obtain that

$$
\frac{|\nabla f|}{1-f} \leq C\left(\frac{1}{R}+\frac{1}{\sqrt{T}}+\sqrt{k}+\sqrt{\widetilde{M}_{\lambda}(-\alpha+1) \tilde{u}_{\min }^{\alpha-1}}\right)
$$

holds at any $(x, t) \in B_{R / 2, T}$, which shows that

$$
\begin{aligned}
\frac{|\nabla u|}{u} \leq & C\left(\frac{1}{R}+\frac{1}{\sqrt{T}}+\sqrt{k}+\sqrt{M_{\lambda}(-\alpha+1) u_{\min }^{\alpha-1}}\right) \\
& \cdot\left(1+\log \frac{B}{u}\right) .
\end{aligned}
$$

Case Three $(\alpha \in(0,1))$. In this case, we also have $e^{(\alpha-1) f}>1$. Hence

$$
\begin{aligned}
2 \tilde{\lambda}\left(\alpha-\frac{-f}{1-f}\right) e^{(\alpha-1) f} \psi w \\
\leq C \frac{\tilde{\lambda}^{2}}{1-f}\left(\alpha-\frac{-f}{1-f}\right)^{2} \tilde{u}_{\min }^{2(\alpha-1)} \\
\quad+\frac{1}{16}(1-f) \psi w^{2} .
\end{aligned}
$$


Putting (59), (26)-(27) into (48), we obtain

$$
\begin{aligned}
& (1-f) \psi w^{2} \leq C\left[\frac{1}{R^{4}} \frac{f^{4}}{(1-f)^{3}}\right. \\
& \left.\quad+\frac{\widetilde{\lambda}^{2}}{1-f}\left(\alpha-\frac{-f}{1-f}\right)^{2} \tilde{u}_{\min }^{2(\alpha-1)}+\frac{1}{R^{4}}+\frac{1}{T^{2}}+k^{2}\right] .
\end{aligned}
$$

Because of $(\alpha-(-f /(1-f)))^{2} \leq 1$, we can obtain that

$$
\frac{|\nabla f|}{1-f} \leq C\left(\frac{1}{R}+\frac{1}{\sqrt{T}}+\sqrt{k}+\sqrt{|\tilde{\lambda}| \tilde{u}_{\min }^{\alpha-1}}\right)
$$

holds at any $(x, t) \in B_{R / 2, T}$, which shows that

$$
\begin{aligned}
& \frac{|\nabla u|}{u} \\
& \quad \leq C\left(\frac{1}{R}+\frac{1}{\sqrt{T}}+\sqrt{k}+\sqrt{|\lambda| u_{\min }^{\alpha-1}}\right)\left(1+\log \frac{B}{u}\right) .
\end{aligned}
$$

We complete the proof of Theorem 2.

\section{Conflict of Interests}

The authors declare that they have no competing interests.

\section{Authors' Contribution}

The authors declare that the work was realized in collaboration with the same responsibility. All authors read and approved the final paper.

\section{Acknowledgments}

The authors would like to thank an anonymous referee for his or her careful reading and helpful comments which made this paper more readable. The research of the first author is supported by NSFC (nos. 11371018 and 11171091), Henan Province Backbone Teacher (no. 2013GGJS-057), and IRTSTHN (14IRTSTHN023). The research of the second author is supported by NSFC (no. 11401179) and Henan Provincial Education Department (no. 14B110017).

\section{References}

[1] G. Y. Huang and B. Q. Ma, "Gradient estimates for a nonlinear parabolic equation on Riemannian manifolds," Archiv der Mathematik, vol. 94, no. 3, pp. 265-275, 2010.

[2] G. Y. Huang and B. Q. Ma, "Gradient estimates and Liouville type theorems for a nonlinear elliptic equation," Archiv der Mathematik, vol. 105, no. 5, pp. 491-499, 2015.

[3] G. Y. Huang, Z. J. Huang, and H. Li, "Gradient estimates and differential Harnack inequalities for a nonlinear parabolic equation on Riemannian manifolds," Annals of Global Analysis and Geometry, vol. 43, no. 3, pp. 209-232, 2013.

[4] P. Li and S.-T. Yau, "On the parabolic kernel of the Schrödinger operator," Acta Mathematica, vol. 156, no. 1, pp. 153-201, 1986.
[5] J. F. Li and X. J. Xu, "Differential Harnack inequalities on Riemannian manifolds I: linear heat equation," Advances in Mathematics, vol. 226, no. 5, pp. 4456-4491, 2011.

[6] L. Ma, "Gradient estimates for a simple elliptic equation on complete non-compact Riemannian manifolds," Journal of Functional Analysis, vol. 241, no. 1, pp. 374-382, 2006.

[7] Y. Y. Yang, "Gradient estimates for a nonlinear parabolic equation on Riemannian manifolds," Proceedings of the American Mathematical Society, vol. 136, no. 11, pp. 4095-4102, 2008.

[8] L. Chen and W. Y. Chen, "Gradient estimates for a nonlinear parabolic equation on complete non-compact Riemannian manifolds," Annals of Global Analysis and Geometry, vol. 35, no. 4, pp. 397-404, 2009.

[9] N. T. Dung and N. N. Khanh, "Gradient estimates of HamiltonSouplet-Zhang type for a general heat equation on Riemannian manifolds," Archiv der Mathematik, vol. 105, no. 5, pp. 479-490, 2015.

[10] S.-Y. Hsu, "Gradient estimates for a nonlinear parabolic equation under Ricci flow," Differential and Integral Equations, vol. 24, no. 7-8, pp. 645-652, 2011.

[11] P. Souplet and Q. S. Zhang, "Sharp gradient estimate and Yau's Liouville theorem for the heat equation on noncompact manifolds," Bulletin of the London Mathematical Society, vol. 38, no. 6, pp. 1045-1053, 2006.

[12] R. S. Hamilton, "A matrix Harnack estimate for the heat equation," Communications in Analysis and Geometry, vol. 1, no. 1, pp. 113-126, 1993.

[13] J. Y. Li, "Gradient estimate for the heat kernel of a complete Riemannian manifold and its applications," Journal of Functional Analysis, vol. 97, no. 2, pp. 293-310, 1991.

[14] X. B. Zhu, "Gradient estimates and Liouville theorems for nonlinear parabolic equations on noncompact Riemannian manifolds," Nonlinear Analysis: Theory, Methods \& Applications, vol. 74, no. 15, pp. 5141-5146, 2011.

[15] Y. Y. Yang, "Gradient estimates for the equation $\Delta u+c u^{-\alpha}=$ 0 on Riemannian manifolds," Acta Mathematica Sinica, English Series, vol. 26, pp. 1177-1182, 2010.

[16] J. Zhang and B. Q. Ma, "Gradient estimates for a nonlinear equation $\triangle_{f u}+c u^{-\alpha}=1$ on complete noncompact manifolds," Communications in Mathematics, vol. 19, pp. 73-84, 2011.

[17] M. Bailesteanu, X. D. Cao, and A. Pulemotov, "Gradient estimates for the heat equation under the Ricci flow," Journal of Functional Analysis, vol. 258, no. 10, pp. 3517-3542, 2010.

[18] S. P. Liu, "Gradient estimates for solutions of the heat equation under Ricci flow," Pacific Journal of Mathematics, vol. 243, no. 1, pp. 165-180, 2009.

[19] J. Sun, "Gradient estimates for positive solutions of the heat equation under geometric flow," Pacific Journal of Mathematics, vol. 253, no. 2, pp. 489-510, 2011.

[20] Q. S. Zhang, "Some gradient estimates for the heat equation on domains and for an equation by Perelman," International Mathematics Research Notices, vol. 2006, no. 1, Article ID 92314, 39 pages, 2006. 


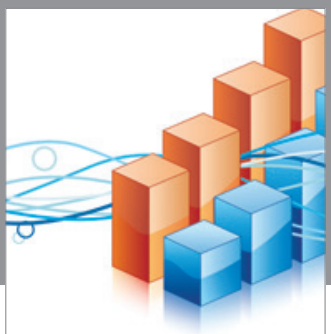

Advances in

Operations Research

vatem alat4

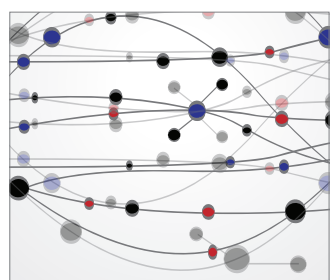

\section{The Scientific} World Journal
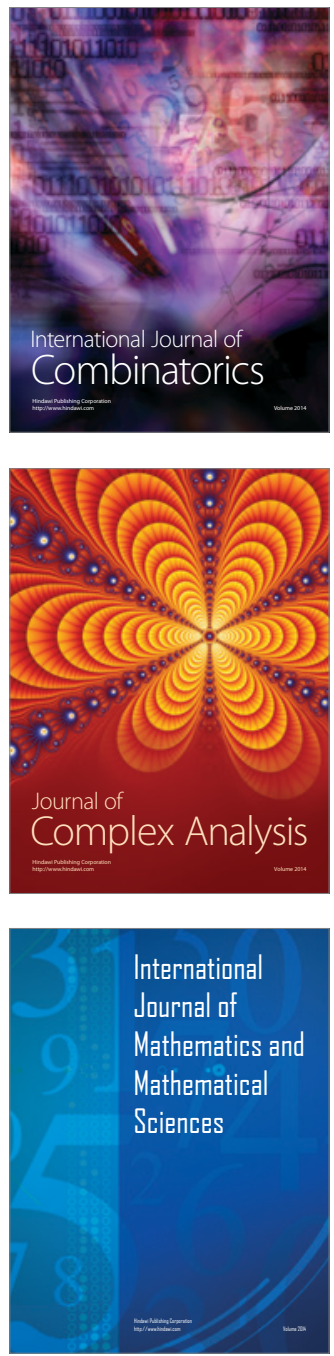
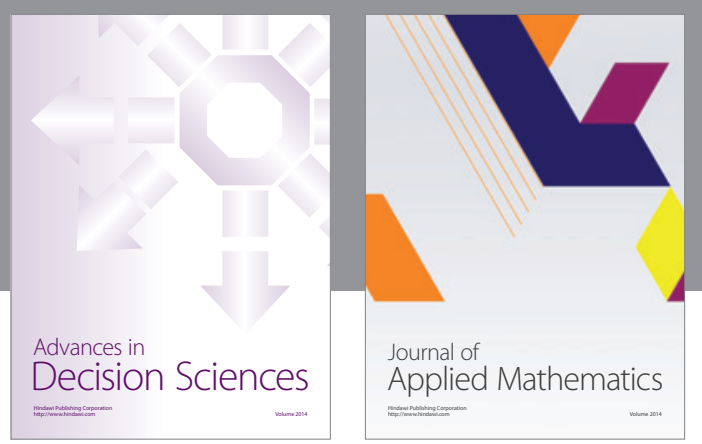

Algebra

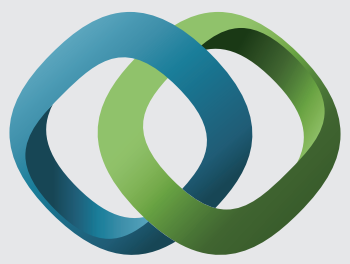

\section{Hindawi}

Submit your manuscripts at

http://www.hindawi.com
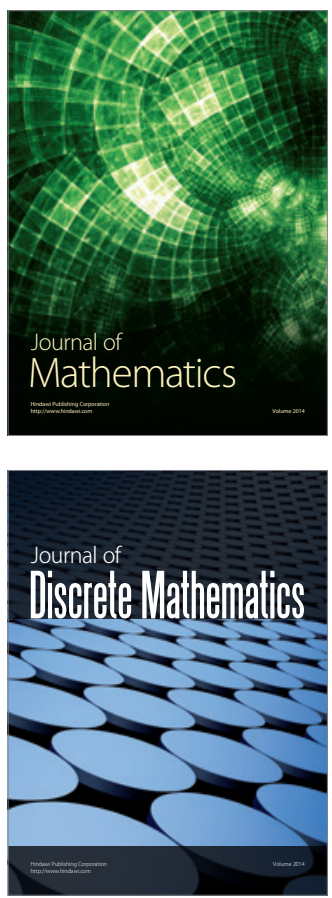

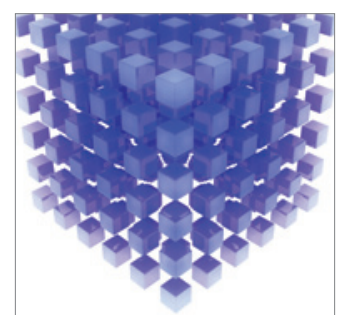

Mathematical Problems in Engineering
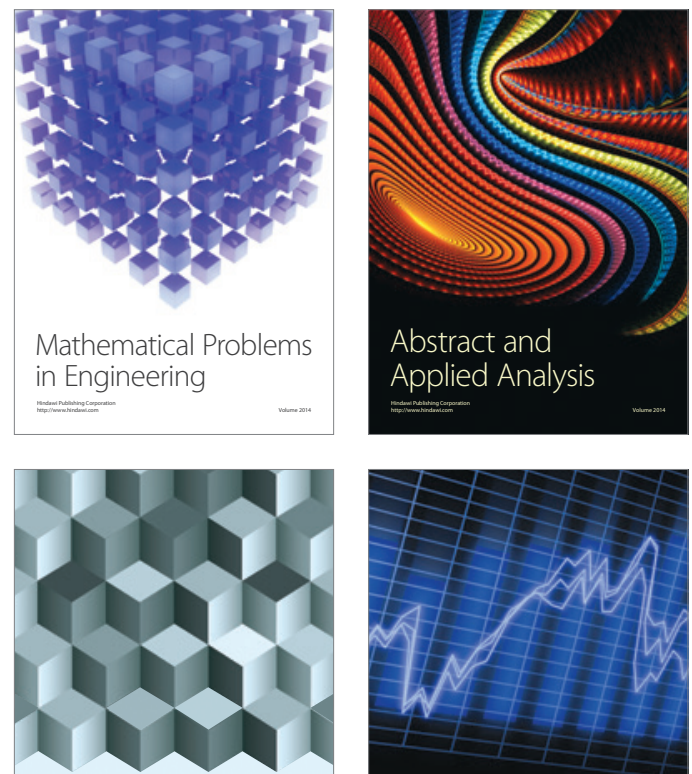

Journal of

Function Spaces

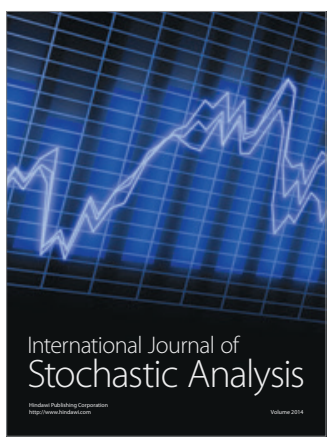

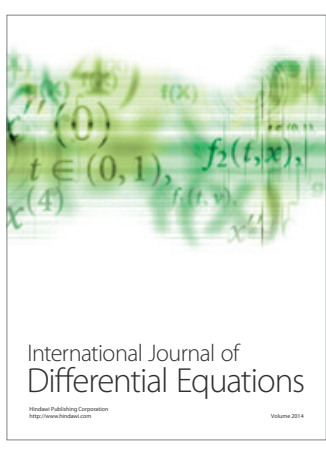
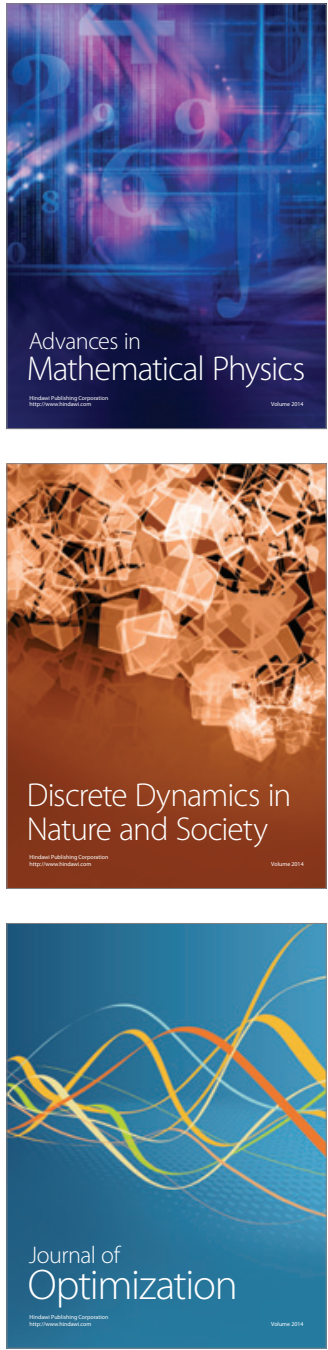